\title{
Analysis of fretting fatigue of late-model flat in the engineering application and research
}

\author{
Wang Yongyan ${ }^{1, \text { a }}$, Zeng Chunlei ${ }^{1, \mathrm{~b}}$, Wu Yang ${ }^{1, \mathrm{c}^{*}}$, \\ ${ }^{1}$ College of Electromechanical Engineering, Qingdao University of Science and Technology, \\ Qingdao 266061, China \\ awangyongyan168@163.com, bzcl82216@126.com, 'c403751612@qq.com
}

Keywords: ANSYS; flat; fretting fatigue; contact stress; finite element method.

Abstract. Applying the ANSYS, the three-dimensional finite element analysis is made on the late-model flat plane-to-plane contact. It also verifies the correctness of the flat fretting fatigue of the finite element analysis by comparing with Mindlin theory. It analyzes the factors such as stiffness coefficient and material properties which influence the flat fretting fatigue, and lays the theoretical foundation to solve wide carding machine flat in the actual plane-to-plane contact.

\section{Introduction}

Fretting refers to relative movements of small amplitude between surface in contact under the effect of alternating load such as mechanical vibration, fatigue load, electromagnetic vibration or thermal cycling (the unit of displacement amplitude is um). Fretting fatigue is fretting, which refers to the relative displacement of the contact surface whose deformation caused by a contact pair enduring alternating stress fatigue and fretting fatigue can accelerate the initiation and propagation of cracks, so that the fatigue life of the components is reduced greatly. Besides, fretting fatigue limit is even lower than the $1 / 3$ of the fatigue limit. Therefore, the fretting fatigue is an important cause of fretting damage. At present, research on the method of fretting fatigue is physical experimental method and numerical analysis method, among which the numerical analysis method has been widely used with its strong adaptability, low cost and short cycle.

In order to better understand the mechanism of action of alternating load between flat and track, the research compared with the Mindlin theory. The results demonstrate the correctness of using ANSYS to analyze fretting analysis of the plane-to-plane contact between flat and track. According to the actual situation has carried on the analysis to the calculation, and obtained some valuable conclusions, thus lay the foundation to improve the life of the flat.

\section{Establishment of finite element model}

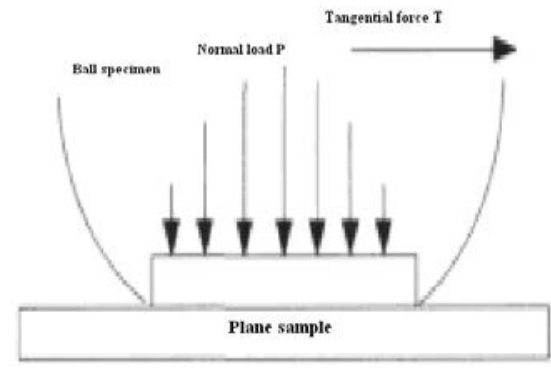

Fig.1 Diagram of ball-to-plane contact

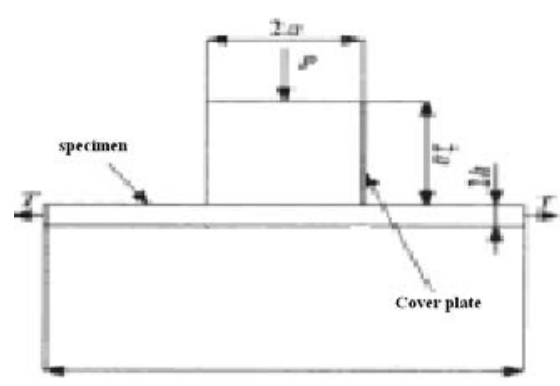

Fig.2 Mechanical model

Theoretical foundation. The method for solving partial slip problem was first proposed by Cattaneo in 1938, and the solution of distribution of the ball-to-plane contact stress was given by Mindlin alone in 1949, the model as shown in fig.1. According to the Mindlin theory, when the contact surface applied alternating tangential force $T(T)<p$, tangential force distribution can be expressed as 


$$
\begin{cases}p(x)=-\mu_{p 0} \sqrt{1-\frac{x^{2}}{a^{2}}} & c \leq|x| \leq a \\ p(x)=-\mu_{p 0}\left[\sqrt{1-\frac{x^{2}}{a^{2}}}-2 \frac{c}{a} \sqrt{1-\frac{x^{2}}{a^{2}}}\right] & c_{\max } \leq|x| \leq c \\ p(x)=-\mu_{p 0}\left[\sqrt{1-\frac{x^{2}}{a^{2}}}-2 \frac{c}{a} \sqrt{1-\frac{x^{2}}{a^{2}}}+\frac{c_{\text {max }}}{a} \sqrt{1-\frac{x^{2}}{c^{2}{ }_{\text {max }}}}\right]|x| \leq c_{\text {max }}\end{cases}
$$

Here $C_{\max }$ can be expressed by equation (2):

$$
\frac{c_{\max }}{a}=\sqrt{1-\frac{T_{\max }}{\mu_{p}}}
$$

And $C$ is $T(T)$ function:

$$
\frac{c}{a}=\sqrt{1-\frac{T_{\max }-T}{2 \mu_{p}}}
$$

Finite element model.We can see from fig.1, the ball-to-plane contact can be understood as a kind of special plane / plane contact. Therefore, we can put the Mindlin ball-to-plane theory to the plane-to-plane stress analysis. Between flat and curved rail eatablished the mechanical model in fig. 2 in this paper. Among them, the compaction force $P$ works on the flat and the lateral alternating force $T$ work on the two ends of flat. Contact surface will produce micro displacement and then exist fretting under the force $P$.The press plate and specimen parameters are as follow: $\mathrm{E}=100 \mathrm{GPa}, \mathrm{v}=0.25$, $\mathrm{a}=100 \mathrm{~mm}, \mathrm{~b}=260 \mathrm{~mm}, \mathrm{~h}=24 \mathrm{~mm}, \mathrm{~d}=20 \mathrm{~mm}$.

According to the structure and the symmetry of the load, we can choose the $1 / 4$ ideal model as simplified model in fig.3. The left $(x=0)$ and the lower side $(y=-h)$ were applied to symmetric boundary conditions and the upper side $(y=2 a)$ and the right $(x=2 b)$ were applied to $p, s$. In order to increase the precision of calculation, we selects 3-d massive element, using Contact Manager tools to generate 3 -d contact pair. Normal contact stiffness and permeability range is 1.0 and 0.1 , $u=0.8$.

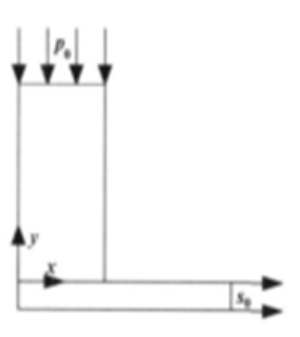

Fig.3 The simplified model

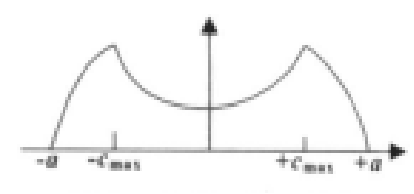

Fig.4 The $\mathrm{S}_{\mathrm{Z}}$ distribution obtained by the Mindlin theory

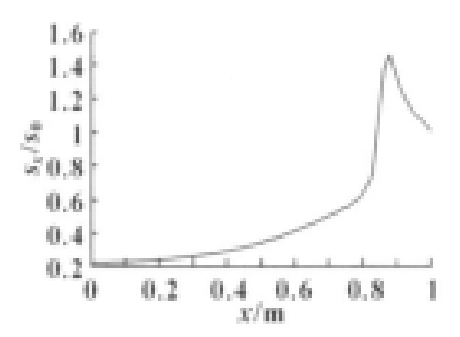

Fig.5 The $\mathrm{S}_{\mathrm{Z}}$ distribution analyzed by finite element

Calculation and comparative analysis. Under the alternating load, we can obtain the distribution of the tangential stress $S_{Z}$ in fig.4 by the formula (1) when $T=T_{\max }$, in adhesive area boundary $\left(x=c_{\max }\right)$ appears singular point of the shear stress, while the shear stress in the gross slip regime is the continuous function of contact position.

Take $S_{0}=P_{0}=10 \mathrm{MPa}$ and obtain tangential stress varying with $\mathrm{x}$ shown in fig.5; compare fig.4 and fig.5 on right section, and we can find two $S_{Z}$ distribution curves match well, which verified the correctness of fretting fatigue analysis by ANSYS. 


\section{The effect of main parameters on the cover of the fretting fatigue}

The stress state of fretting contact area between flat and track is the main factor to cause and deepen the fatigue crack. The fretting fatigue cracks was firstly observed between the stick contact zone near the junction and the contact surface tilt direction. By analyzing the stress field, the initiation of the inclined crack could be formed due to synthetic shearing force of the tangential stress and the macroscopic axial stress. Under cyclic loading, contact interface appeared variable stick contact area and its tensile stress distribution state of internal alternating controlled on the crack early expansion, when the inclined crack propagation was far from a certain distance from the fretting surface, tensile stress will be substantially reduced, and the axial cyclic stress paralleling to the contact surface plays a dominant role, leading to crack propagation transforming from type II to type I. Therefore. It is the key to analyzing the distribution of contact stress between flat and track on the fretting fatigue of quantitative study.

Effects of stiffness on the fretting fatigue of flat. The fretting displacement amplitude is usually in micrometer. The magnitude is equal to the contact surface of the elastic deformation, so the flat stiffness has a great influence on the variation characteristics of fretting fatigue. We could obtain the stiffness $K$ from friction-displacement curve in the fretting experiment, which is the comprehensive result of the contact stiffness $K_{c}$ and system stiffness $K_{s}$, namely:

$$
\frac{1}{K}=\frac{1}{K_{C}}+\frac{1}{K_{S}}
$$

$K_{c}$ depends on the material properties and contact load while it is set artificially in finite element analysis. $K_{s}$ is the comprehensive effects of various stiffness (including clamping mechanism, pattern geometry and installation conditions).

In order to study the influence of stiffness coefficient on contact load, with other parameters unchanged, the normal contact stiffness were set 0.5,1.0,1.5 when forming contact pairs to calculate respectively, so the Sz Sy reached the distribution of the maximum amplitude in alternating load in fig.6-8.

When specimens are under tensile stress, Sz varies with the distribution of stiffness coefficient in fig.6.With the increase of stiffness coefficient, the same value $\mathrm{x}$ corresponding to the Sz value decreases, the reason is that the stiffness is increased and elastic deformation of the contact surfaces decreased, thus reducing the stress value; along with the increase of stiffness coefficient, stress change relatively early in the junction mixed regime and slip regime, namely the slip area increasing, the reason is that with its increasing the stiffness coefficient under given alternating load between the contact surfaces are easier to slip.

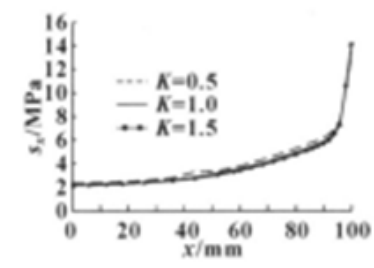

Fig.6 The Sx curves of different stiffness coefficients(T)

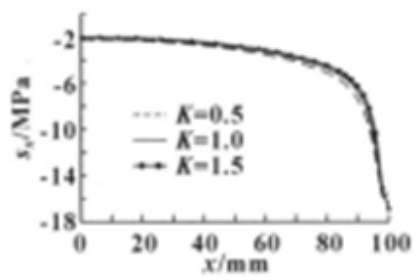

Fig.7 The Sz curves of different stiffness coefficients(T)

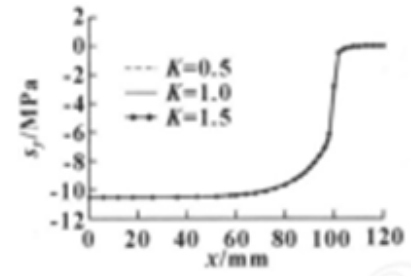

Fig.8 The Sy curves of different stiffness coefficients(T)

When specimen are under pressure stress, Sz varies with the distribution of different stiffness coefficient in fig.9, with the increase of stiffness coefficient, the same value x corresponding to the $\mathrm{Sz}$ values increased, but the absolute value decreases.

When specimen are under tensile stress, Sy varies with the distribution of stiffness coefficient in fig.8. The three curve Sy coincides basically, so stiffness coefficients has little influence on the 
distribution of Sy. Besides, the Sy reflects the normal contact condition of the two contact surface. It could be argued that variation of stiffness coefficient has no impact on the whole contact region.

Sum up and draw the conclusion: with stiffness coefficient decreasing, $\Delta \mathrm{S}_{\mathrm{Z}}\left(\Delta \mathrm{S}_{\mathrm{Z}}=\mathrm{St}-\mathrm{Sp}\right)$ increasing and Sy basically being unchanged, the ratio of flat and track contact mixed zone in the whole fretting area increased, the size of the whole fretting region does not change. As the contact mixed zone is the main zone for micro crack to produce and expand rapidly, thus the increasing of mixed zone and value $\Delta \mathrm{S}_{\mathrm{Z}}$ accelerated fretting fatigue, reduced the service life of the flat.

\section{Effect of Material Properties on Fretting Fatigue}

Take cast iron as material above before improvement $(E=150 G P a, V=0.25)$. To analyze the influence of material properties on the fretting fatigue, we take 2091 aluminum alloy $(E=70 G P a, V=0.3)$, alloy steel $(E=206 G P a, V=0.3)$ and cast iron respectively to form a new experimental model. With parameters unchanged, take flat/track casting as aluminum/cast, steel/aluminum, steel/ cast and cast/steel respectively and called scheme 1, scheme 2, scheme 3 and scheme 4 respectively. In order to obtain more obvious effect, we take $S_{0}=10 M P a, P_{0}=2 M P a$ to calculate each scheme. So that the $\mathrm{Sz}, \mathrm{Sz}$ distribution when tensile-press stress reaching peak are shown in fig.9 and fig. 10.
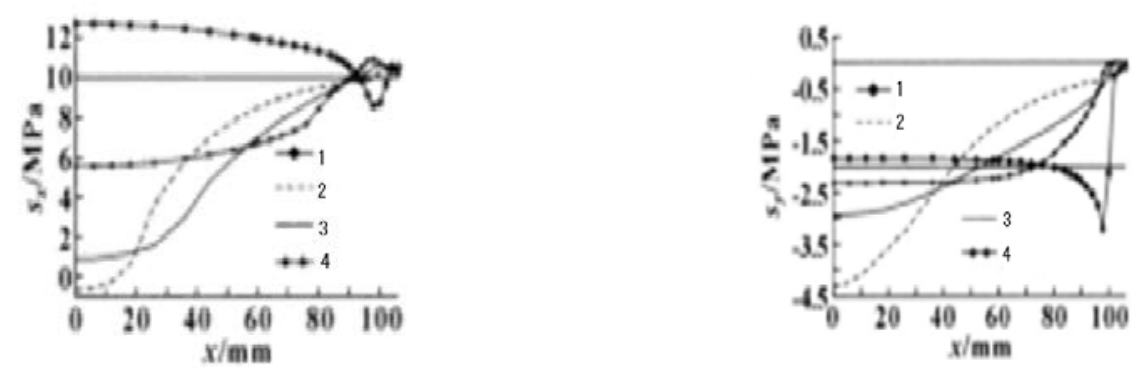

Fig.9 Different schemes of Sz, Sy distribution(T)
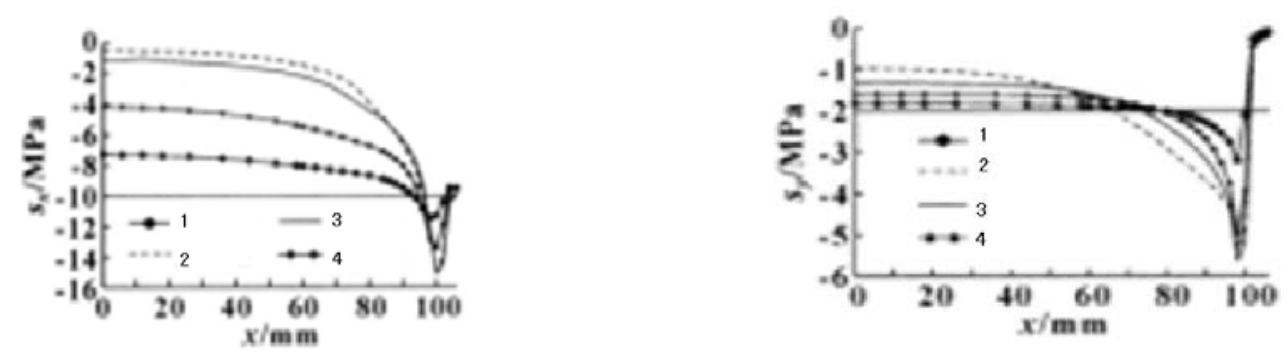

Fig.10 Different schemes of Sz, Sy distribution(P) 


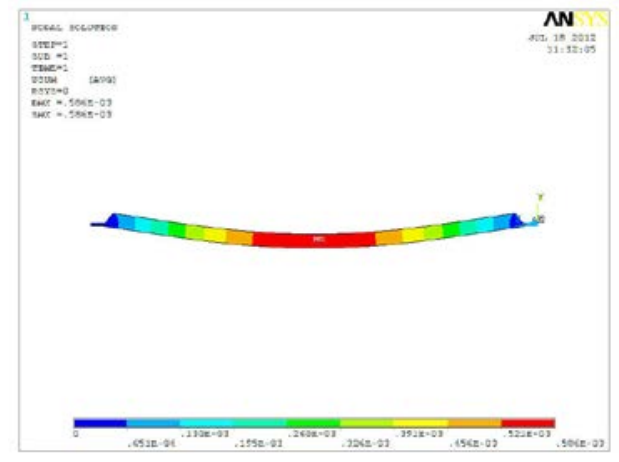

Fig.11 The overall displacement nephogram when aluminum alloy flat $\mathrm{T}=\mathrm{Tmax}$

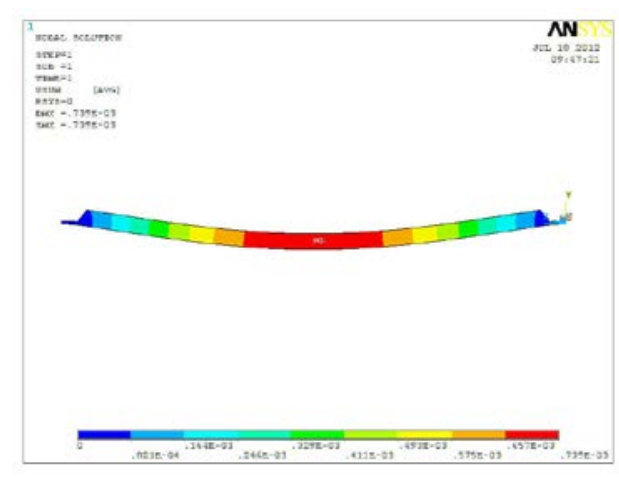

Fig.13 The overall displacement nephogram when cast iron flat $\mathrm{T}=\mathrm{Tmax}$

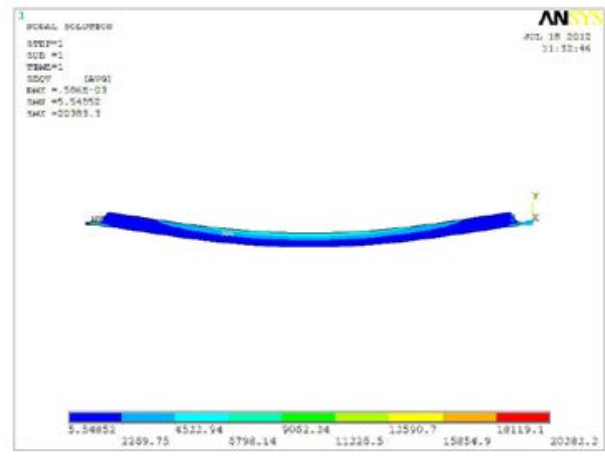

Fig.12 The overall equivalent stress nephogram when aluminum alloy flat $\mathrm{T}=\mathrm{Tmax}$

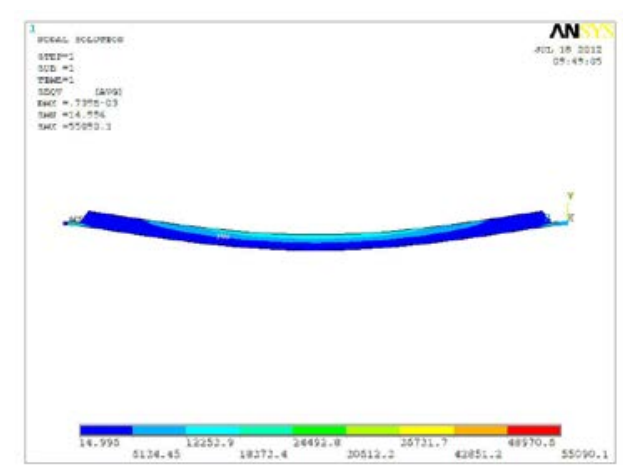

Fig.14 The overall equivalent stress nephogram when cast iron flat $\mathrm{T}=\mathrm{Tmax}$

Under alternating load select three materials, i.e. aluminum alloy, cast iron and alloy steel and obtain the two kinds of nephograms shown from fig.11 to fig.14 by analysis.

Different schemes have different stress distribution and displacement in the figure. However, what is common is that there exists a instable contact region on the edge of fretting contact region within the existing great stress vibration, where is a mixed adhesive-sliding zone, and is expanded gradually with the fretting time increasing, eventually causing fretting damage.

Comparing scheme 1 with 3 , as the elastic modulus of aluminum alloy is smaller, there is a great elastic deformation making the decrease in the bound effect between track and flat and also a great deformation on the tensile effect. It was more likely to occur fretting fatigue on the edge of contact zone compared scheme 3 with 1 in $\Delta \mathrm{S}_{\mathrm{Z}}$.

Comparing scheme 2 with $3, \mathrm{~S}_{\mathrm{Z}}$ and Sy presented the same trend: the elastic modulus of track is lower than flat's. The track has a strong bound effect on flat from the curve $\mathrm{S}_{\mathrm{Z}}$. The difference is that the elastic modulus of cast iron is larger than 2091 aluminum alloy's. The track has little bound effect on it. The mixed area of scheme 3 is larger and is easy to cause fretting damage.

Comparing scheme 3 with 4 , when it's near the center, $S_{z 3}<S_{z 4}$. When it's in the edge, $S_{z 4}<S_{z 3}$. sy approaches 0 early in scheme 4 , namely the flat and track are early to open on the edge of contact. The bound ability in scheme 3 is obviously better than 4 , which can effectively decrease the damage caused by fretting fatigue.

Comparing scheme 1 with 4, aluminum alloy could absorb part or evem all of the fretting amplitude by the elastic deformation of contact surface, making the fretting be in elastic regulation state, namely the operation in the partial slip zone.

The ratio of the elastic modulus between briquette and specimen is $\lambda$, so they are $\lambda_{1}, \lambda_{2}, \lambda_{3}, \lambda_{4}$ in the four schemes and $\lambda_{2}>\lambda_{3}>\lambda_{4}>\lambda_{1}$ in calculation. With the comparison, we found that the bound ability of track reduced with $\lambda$ decreased, the flat is easy to strain and occur fretting fatigue 
and damage under fatigue load. But if elastic modulus of the track decreases to a certain degree, it is beneficial to absorb relative sliding and prevent fretting.

\section{Summary}

By calculating each scheme with analysis, we can obtain the following conclusions:

(1) Using ANSYS to analysis fretting fatigue is correct.

(2) Three-dimensional block element method of fretting fatigue problems is feasible, the result is closer to the actual situation.

(3) With the stiffness coefficient decreasing, the mixed zone increases, and fretting fatigue intensifies within a certain range; stiffness coefficient has little effect on the whole contact area.

(4)The reasonable choice of contact materials and matching have an important influence on alleviating fretting damage. Good flexible and large deformation material can effectively absorb the relative sliding under the condition of meeting intensity, so as to reduce the surface damage. Material with high hardness and fatigue strength can effectively reduce the fretting wear and inhibit the initiation and extension of crack.

\section{Acknowledgement}

Special thanks to Mechanics Research Center, Qingdao University of Science \& Technology, which provide me with all conditions and facilities for this experiment.

I gratefully acknowledge the financial support from the National Natural Science Fund project (51374134), the Chinese Education Ministry doctoral program project (20133719110005), and the Natural Science Foundation of Shandong Province (ZR2014EL20), and the Qingdao basic research project of science and technology program(12-1-4-3-(12)-jch).

Corresponding author: Wu Yang

Corresponding method: 403751612@qq.com 15165266296

\section{Reference}

[1] Zhongrong Zhou, Minhao Zhu. Complex fretting wear of [M]. Shanghai: Shanghai Jiao Tong University press, 2004.

[2] Yuan Wang, Ridong Liao, Limin Yao. Finite element analysis of fretting action on component [J]. strength and environment, 2005, 324: 27 - 32.

[3] R.B. Waterhouse. Fretting fatigue [M ]. London: Applied Science, 1981.

[4] R.D. Mindlin. Compliance of elastic bodies in contac [J]. ASME Journal of Applied Mechanics, 1949, 16: 259 - 268.

[5] Hua Zhao, Zhongrong Zhou. The progress of application on numerical methods in the fretting fatigue research [J]. tribology, 2000, 204: 317 - 320. 